\title{
PRA EFATIO.
}

Decimus quintus hic annus est, quum editis Philemonis et Menandri reliquiis graecorum comicorum fragmenta uno corpore comprehensa me editurum esse publice professus sum * * Erat tum ea temporum meorum opportunitas, ut quamvis magnum opus et in varias antiquitatis partes diffusum intra quinquennium rite absolvi posse confiderem. At vero quum iam in digerenda, quan undique collegissem, materia ! occupatus essem, eae subito rerum mearum vicissitudines inciderunt, quae me in proposito opere mirifice retardarent. Nam quum Gedani, quae mihi

*) Idem consilium superiore aetate agitasse T. Hemsterhusium colligere licet ex is quae scripsit ad Polluc. vol. II p. 1136. Theodori Canteri syllogen comicorum et tragicorum poetarum manuscriptam commemorat $H$. Grotius Praef. Excerpt. p. 8 et D'Orvillius Vanno crit. p. 251. De Bentleii omnium graecorum poetarum fragmenta colligendi consilio vide Valckenarium Diatr. p. 4 A. 
quasi adoptiva patria fuit, inter iucundissimas publici muneris occupationes satis otii ad haec studia colenda et doctrinam augendam mili concessum fuisset, mox Berolinum vocatus et publicarum et privatarum curarum quasi aestu quodam adeo me iactatum sensi, ut satis mihi beatus viderer, si subingde velut furtivas horas gravioribus negotiis ereptas coepto operi impendere liceret. Accedebat, ne inexpugnabilem illam ingenii mei ad scribendum tarditatem commemorem a prompta multorum alacritate vehementer discrepantem, iusta quaedam, ut mihi videbatur, et litterarum dignitati debita verecundia, qua accuratam et diligentem quam levem et praecipitem curam in suscepto negotio collocare malebam. Etsi enim in tali labore plenam omnium rerum pertractationem iure exspectari non posse intelligebam, vehementer tamen animus ab eorum festiuatione et levitate abhorrebat, quos necessariam aedificio struendo materiam raptim ex indicibus colligere et properatos ingenii foetus in publicam lucem protrudere videbam. Itaque lente quidem nec sine maguis quibusdam commentandi intermissionibus, at progrediendo tamen eo perducta res est, ut vetus illud meum consilium iam tandem ad effectum adducere liceat. Erit autem totius operis dispositio haec, ut quum in prima, quam nunc ipsum publici iuris facio, parte poetarum historiam enarraverim, in altero, quod intra proximum anuum sequetur, volumine antiquorum, in tertio mediorum, in quarto denique et quinto volumine novorum et anonymorun comicorum reliquiae cum indice grae- 
citatis locupletissimo proponantur. Novae comoediae auctores quod duo volumina impleturos esse dixi, non mirabuntur, opinor, qui Philemonis et Menandri fragmenta, quae ex quo prinum a me edita sunt ingenti numero locupletari multisque partibus emendari possunt, ad integritatem operis omitti non posse sibi persuaserint. Eandem ob causam etiam Aristophanis fragmenta adiicerem, nisi falcem in alienas messes immittere iniquum ducerem.

Iam ut de argumento praesentis huius voluminis quae dicenda sunt paucis complectar, versatur id totum in explicanda poetarum quorum deinceps fragmenta sequentur historia, quam quod criticam dicere ausus sum, vehementer vereor ne aliquanto plus quam efficere potui promisisse videar. Obversabatur autem animo meo forma et descriptio operis fere ea, quam in explananda oratorum historia praeivit Ruhnkenius, ad cuius exempli praestantiam si peritis iudicibus non infeliciter adspirasse visus fuero, satis magnum impensae operae fructum me tulisse arbitrabor. Ceterum meminerint si qui haec lecturi sunt haud exiguam partem eorum, quae hoc volumine continentur, primum in programmatis scholasticis propositam esse, iam inde ab octodecim annis per varias scholasticorum solennium opportunitates evulgatis. * Quibus quidem scriptionibus illud certe mihi conse-

-) Commentationum miscellanearum Fasciculus I prodiit anno MDCCCXXIl, Quaestionum senicarum Specimen primum MDCCCXXVI, secundum MDCCCXXVII, tertium MDCCCXXX. 
quutus esse videor, ut nostratium studia ad hanc fructuosissimam sed fere neglectam et senescentem graecae antiquitatis partem converterim, meoque exemplo excitati complures in eodem argumento sibi elaborandum esse decreverint, vario illi quidem successu, neque omnes ita ut operae pretium fecisse existimandi sint. At magna cum laude commemorandus mili est Theodorus Bergkius, cuius doctissimae commentationes de reliquiis comoediae atticae antiquae, quamquam haud pauca habent in quibus mili dissentiendum esse video a viro carissimo, plurimas tamen observationes continent ad explicandam antiquae comoediae rationem utilissimas. Quo libro si uti mihi licuisset in is praesertim, quae de antiquae comoediae poetis commentatus sum, pluribus de rebus et copiosius et vero etiam aliquanto rectius disserere licuisset. Nuuc ille eo demum tempore ad me perlatus est, quo primam scriptorum partem librario typis imprimendam ian tradideran. Versantur autem Bergkii commentationes maxime in explicandis Cratini comoediis, quae res quum aute hos duos ferme annos, quo tempore ego quaestiones meas retractare coeperam, ab ipso mihi per litteras significata esset, in mea de illo poeta disputatione, qualem in Quaestionum scen. spec. I exhibui, praeter minutiora quaedam nihil mutare satius duxi.

Ceterum quod plerisque scribentium accidere solet, ut, quum ad extremam manum perventum fuerit, eorum quae scripserunt fere eos poeniteat, id mihi adeo usque accidisse fateor, ut, si fieri posset, 
magnam imo vero maiorem libri partem iteratis curis refingerem. Sed facta infecta reddi non possunt: cui incommodo ut quantum in me positum esset subvenirem, appendicem additurus eram, in qua falsa corrigerem, omissa supplerem, alia de quibus in utramque partem disputari posset retractarem. Sed quum in ceteris voluminibus non benigniorem mili sortem fore praevideam, optimum arbitror, ne ,addenda" atque ,etiam addenda" per singulas deinceps partes sese excipiant, quidquid vel corrigendum vel supplendum videbitur in calcem totius operis reiicere. Quam ob causam angustis nunc quidem me continebo terminis, ea tantum hoc loco commemoraturus, quibus quod superest chartae haud inutiliter impleri posse videbitur.

Primum igitur manifesto errore lapsus sum p.410, ubi incertae Aristophontis fabulae fragmentum servatum esse dicebam a Theophilo. Sequutus tum eram H. Grotii coniecturam, qui Excerptis p. 1004 A ristonis nomini Aristophontem substituit. Theophili locus in libro ad Autolycum III p.296 hic est:

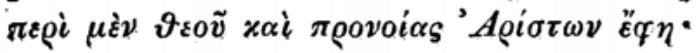

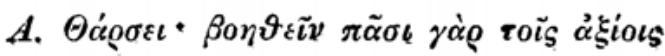

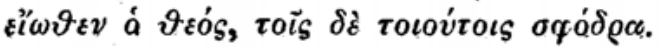

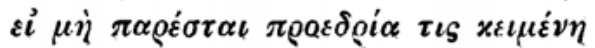

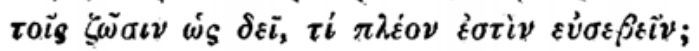

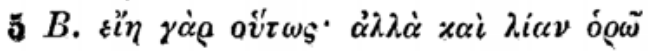

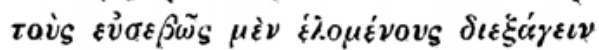

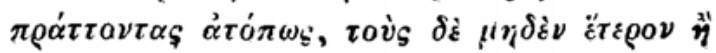

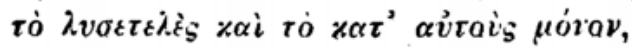

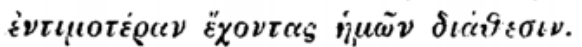




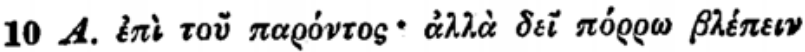

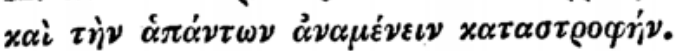

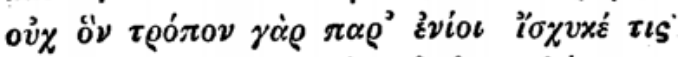

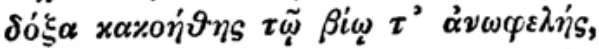

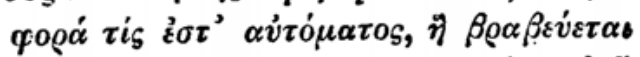

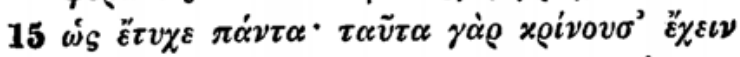

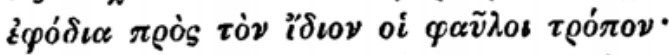

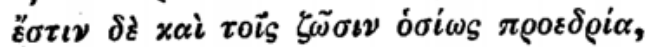

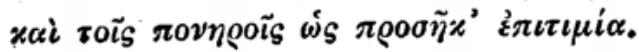

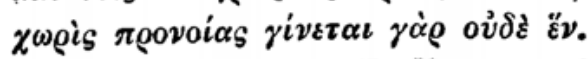

Vs. 1 rà $\varrho$ abest a codice Bodl. et antiquis editioni-

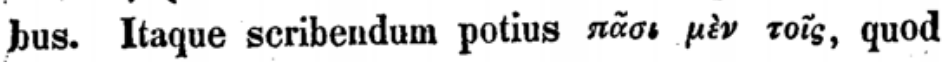
etiam sequentia commendant. Vs. 3 scribendum est

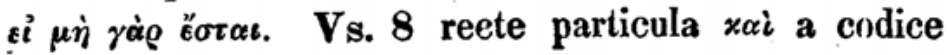
Bodl. omittitur. Probabile est enim scriptum fuisse

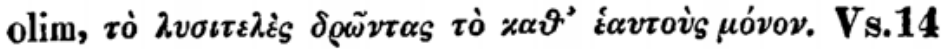
insolens est $\beta \rho \alpha \beta \varepsilon \dot{v} \varepsilon \sigma \vartheta \alpha \iota$ potestate activa. Fortasse

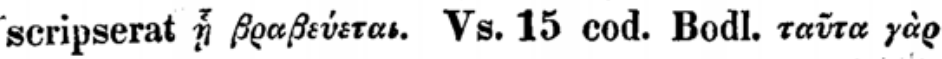

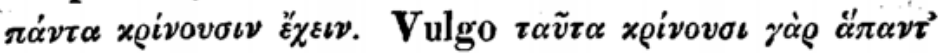
Ě $\chi s ı v$. Retinui Grotii coniecturam, quamquam ne ita quidem locum intelligo. Hi igitur versus mili quidem neque Aristophontis neque alius cuiusquam poetae comici, sed Aristonis Chii esse videntur, communem Stoicorum morem sequnti, qui decreta sua, maxime ea quae ad morum doctrinam spectarent, iambicis versibus iisque fere ad comicorum numerorum similitudinem effictis includebant, eosque versus ornatus causa reliquae orationi aduniscebant. Ex hoc genere ut pauca de multis afferam est Zenonis illud apud Stobaeum Floril. XIV 4:

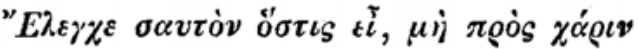

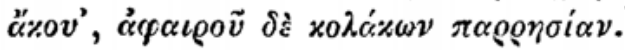


Item Crantoris *) apud eundem XCVI 13:

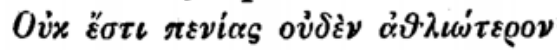

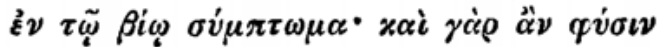

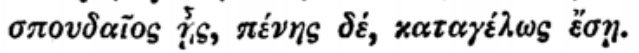

Et eiusdem XCVII 6:

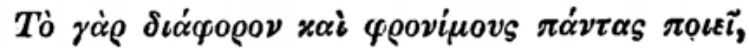

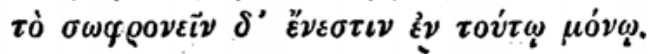

Eadem Chrysippi fuit consuetudo, quamquam is quidem alienos potius maximeque Euripideos quam suos ipsius versus orationi inseruit. V. Diogenes Laert. VII 108. Neque in scriptis tantum sed etiam in scholis hanc rationem tenuerunt philosophi. Cicero Tusc. II 11. Fuisti saepecredo, cum Athenis esses, in scholis philosophorum. A. Yero, ac libenter quidem. M. Animadvertebas igitur, etsi tum nemo erat admodum copiosus, verum tamen versus ab his admisceri orationi. A. Ac multos quidem a Dionysio Stoico. M. Probe dicis; sed is quasi dictata, nullo delectu, nulla elegantia: Philo noster et proprium numerum et lecta poemata et loco adiungebat. Prae ceteris autem Cleanthes eiusmodi flosculis orationem distinguebat, quem quidem etiam hexametris eam in rem usum fuisse intelligitur ex Clemente Alex. Strom. V

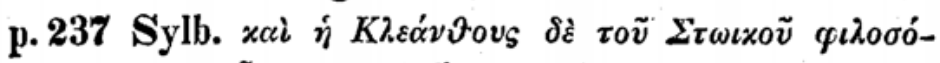

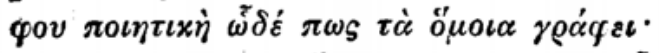

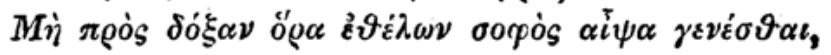

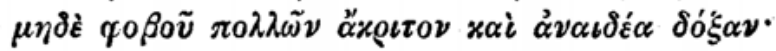

*) Ita recte Grotius. Gesnerus et Gaisfordius $K \varrho \alpha \dot{\nu} \tau \tau \omega \nu$ s. 


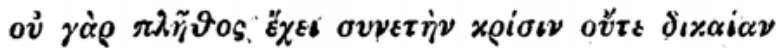

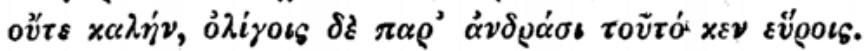
Cuius poematii secundo versu inepte $\delta \dot{\prime} \xi \alpha$ vocatur

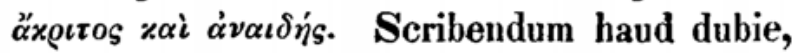

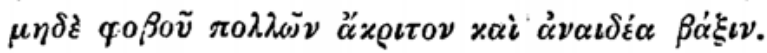

Frequentius tamen etiam Cleanthen iambicis versibus usum esse fragmenta docent, ex quibus ea tantum afferre placet, quae, ut in Aristonis loco factum videmus, colloquentium personarum vicibus distincta sunt, ut etiam hac forma philosophos illos usos esse incelligatur. Galenus de Platone et Hippocr. vol. I

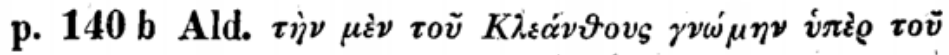

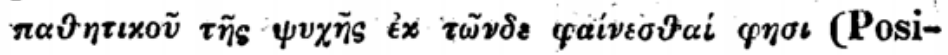
donius) $\tau \tilde{\omega} \nu \dot{\varepsilon} \pi \tilde{\omega} \nu$.

1. T'i $\pi 0 \vartheta$

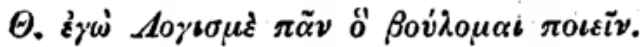

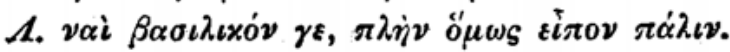

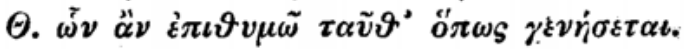

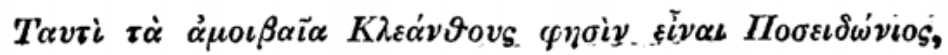

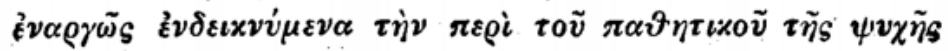

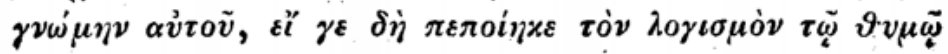

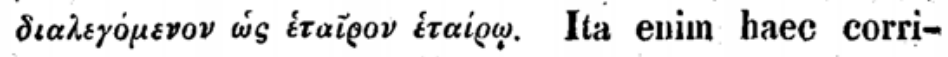
genda videntur. Vulgo in Galeni verbis legitur

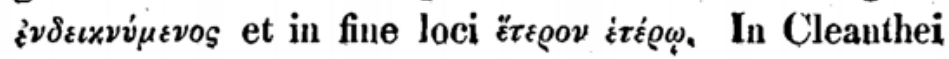
loci versu secundo vulgo est $\lambda o \gamma \iota \sigma \mu o ́ \nu$, correctum a Wyttenbachio ad Posidonium I. Bakii p. 285, in ceteris tamen non audiendo. Sententia eadem esse debet quae quarto versu, ubi $\omega^{\gamma} v$ scripsi pro $\omega$ s, dilucidius explicatur. Versu tertio addidi vai, Ex dialogo item petita videri possunt haec eiusdem Cleanthis apud Stobaeum Flor. VI 20 servata: 


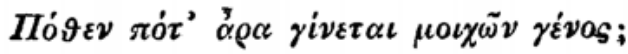

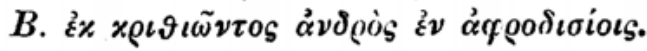

Et quae leguntur apud Clementem Alex. Strom. V

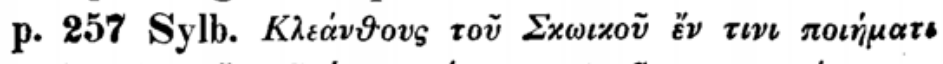

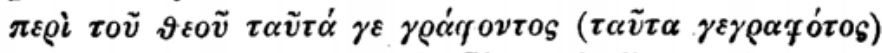

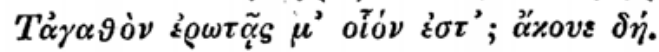

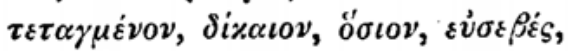

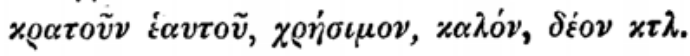

Affinis huic de Aristophonte errori est is quem in iis quae p. 424 de Simylo disserui a me admisissum sero animadverti. Plane enim non dubito quin Simylus is, cuius aliquot fragmenta ex Stobaeo, Ioanne Damasceno et Theophilo exsignavi, a comico eiusdem nominis poeta diversus habendus sit, qua de re quominus dubitari possit ipsos versus adscribam. Stobaeus igitur Floril. LX 4 hunc Simylo locum tribuit:

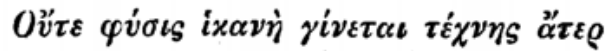

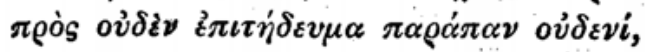

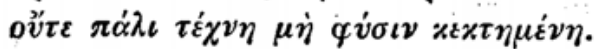

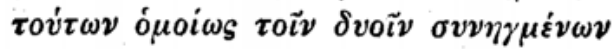

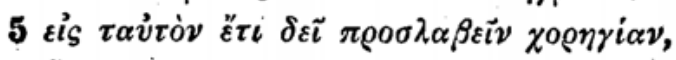

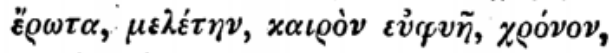

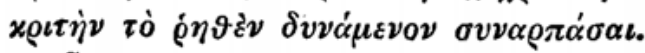

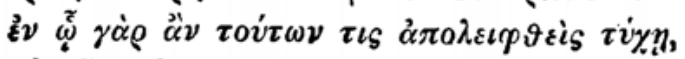

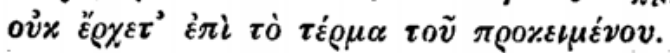

$10 q \dot{v} \sigma \iota_{S} \vartheta \dot{\varepsilon} \lambda \eta \iota_{S} \dot{\varepsilon} \pi \iota \mu \dot{\lambda} \lambda \varepsilon \iota^{2} \varepsilon \dot{v} \tau \alpha \xi \xi i \alpha$

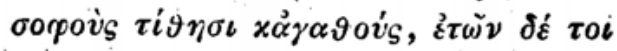

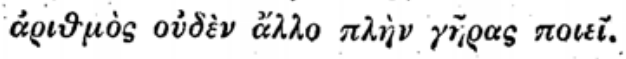

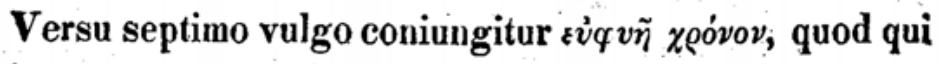
in zogòv mutatum ivit Dobraeus ad Porsoni Aristophanica p. (104), non videtur sententiam loci perspe- 
xisse; nec quinto versu zoønyic de apparatu scenico intelligendum, sed figurato sensu, ut saepe alias, accipiendum est. Alterum huius poetae locum servavit Io. Damascenus. Eum e codice Florentino primus edidit Wyttenbachius ad Plutarchum vol. I p. 45, postea Gaisfordius Appendice ad Stobaeum vol. IV p. 433 ed. Lips. Est autem hic:

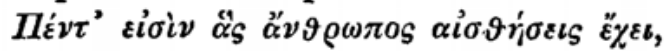

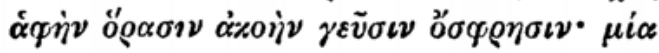

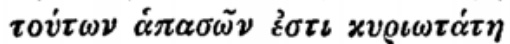

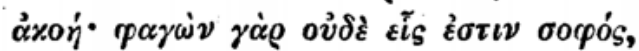

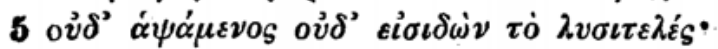

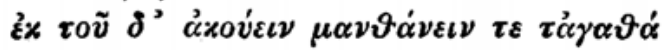

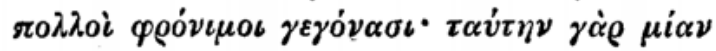

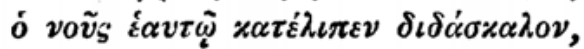

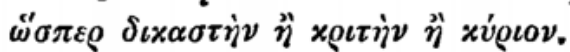

Versu secundo fortasse mutato verborum ordine scribeudum est,

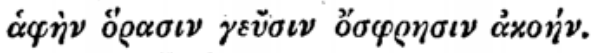

$\tau o v i \omega \nu \mu i^{\prime} \dot{\alpha} \pi \alpha \sigma \omega \tilde{\nu} \chi \tau \lambda$.

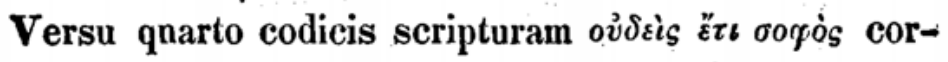
rexi Quaest. scen. III p. 59. Versu quinto sequutus sum Iacobsium Lectt. Stob. p. 152. Codex oủ szis ¿ $\delta \omega \dot{\nu} \tau \varepsilon \lambda v \sigma \iota \tau \varepsilon \lambda \dot{\varepsilon}$. Tertius denique locus, quem eidem Simylo adsignabam, legitur apud Theophilum ad Autol. III p. 385 : $\tau \dot{\nu} \nu$ ơ้

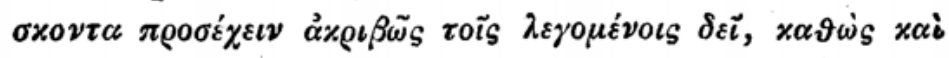

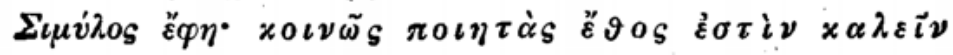

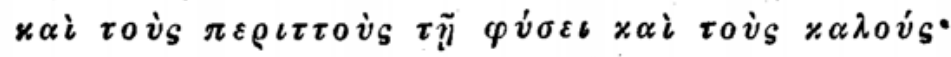
$\varepsilon \delta_{\varepsilon \iota} \delta \dot{\varepsilon} \times \rho i \nu \varepsilon \iota \nu$. Quae cum primo fragmento argumenti affinitate coniuncta asse et ita redintegranda videntur : 


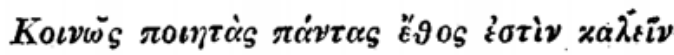

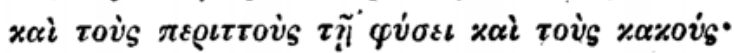
$\ddot{\varepsilon} \delta \varepsilon \iota \delta \xi \varepsilon$ «

Haec igitur fragmenta qui inter se contulerit etargumentum eorum paullo diligentius consideraverit, omnia ex eodem carmine didactico petita, a comoedia autem maxime aliena esse atque omnino florentibus attici sermonis temporibus scribi non potuisse intelliget. Neque improbabile mihi videtur auctorem illius carminis eundem esse Simylum cuius aliquot de Tarpeia versus elegiacos servavit Plutarchus in vita Romuli cap. 17.

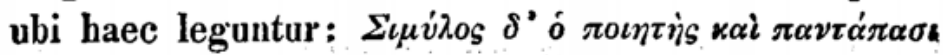

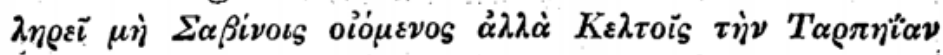

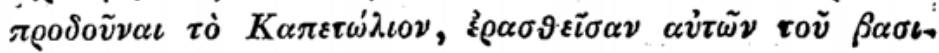
$\lambda \dot{\varepsilon} \omega \mathcal{S} . \lambda \dot{\varepsilon} \gamma \varepsilon \iota \delta \dot{\varepsilon} \tau \alpha \tilde{v} \tau \alpha \cdot$

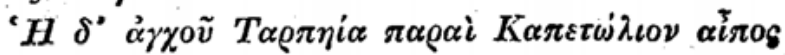

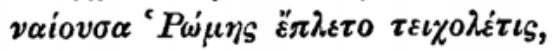

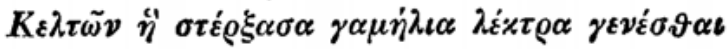

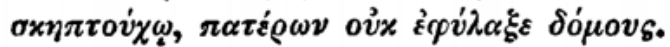

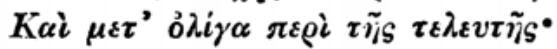

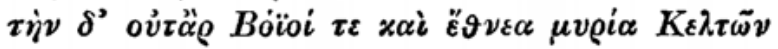

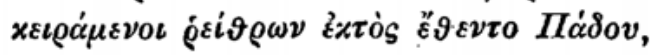

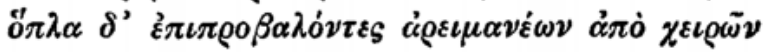

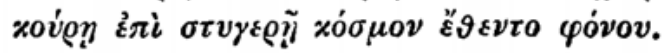

Quae auctorem produnt Augusteo saeculo vix superiorem. Ad iambicum Simyli carmen ut redeam, ipsa orationis ratio satis manifesta habet non atticae originis vestigia: ex quo genere est productio primae syllabae vocabuli $\tau \dot{\varepsilon} \chi \nu \eta$, correptio articuli $\tau \dot{o}$ ante $\dot{\emptyset} \eta \vartheta \dot{\varepsilon} \nu$, accusativus $\varepsilon \dot{v} \varphi v \tilde{\eta}$ pro $\varepsilon \dot{v} \varphi v \tilde{\alpha}$, usus particulae $\pi \dot{\alpha} \lambda \iota$ pro $\pi \dot{\alpha} \lambda \iota v$, qua forma constanter abstinuerunt poetae attici. Unde apertum est non recte nuper virum 
doctissimum in Platonis versu a Scholiasta Veneto ad Aristophanis Pac. 72 servato scripsisse,

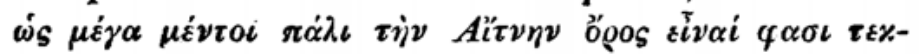

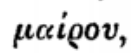

pro eo quod codex babet $\pi \dot{\alpha} \lambda \alpha \iota$, quod in $\pi \dot{\alpha} \nu v$ mutalldum erat. Denique eodem pertinet correptio primae syllabae adiectivi $\lambda v \sigma \iota \tau \varepsilon \lambda \dot{\eta}$, qnam produci et analogia et usus postulat. Ac potest sane etiam Simyli versus facili opera ad communem normam revocari scri-

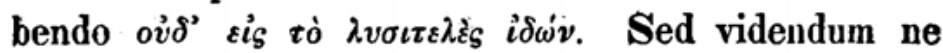
suus Simylo error relinquendus sit, si quidem minus accuratos poetas $\lambda v \sigma \iota t \varepsilon \lambda \dot{\eta} s$ prima brevi extulisse ex Pho-

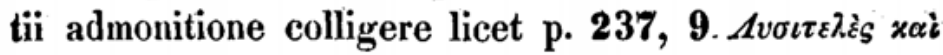

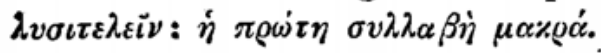

Scribebam Berolini Calendis Decembribus a. MUCCCXXXVIII. 